\author{
Research Report Poster Presentation \\ Number: RR-PO-22-02-Mon \\ Monday 4 May 2015 13:00 \\ Exhibit halls 401-403
}

\section{EXERCISE PRESCRIPTION TO PREVENT INJURIES DURING RECREATIONAL ALPINE SKIING AND SNOWBOARDING}

$\underline{\text { K. Hebert-Losier }}^{1,2}$, H.-C. Holmberg ${ }^{1}$

${ }^{1}$ Mid Sweden University, Health Sciences, Östersund, Sweden; ${ }^{2}$ National Sports Institute of Malaysia, Kuala Lumpur, Malaysia

Background: Skiing and snowboarding are popular recreational sports worldwide, promoting health through physical activity. Regrettably, participation in snow sports also involves injury risk. Each year, skiing and snowboarding activities contribute to the total number of sports-related injuries, and associated high socio-economic and personal costs. In several sports, targeted exercise programs are reported to be effective in reducing injury rates, and therefore have the potential to prevent skiing and snowboarding injuries.

Purpose: The purpose of this work was to systematically review the literature to identify exercises and/or training strategies that are suggested to prevent alpine skiing and snowboarding injuries in recreational participants.

Methods: Fourteen electronic databases were systematically searched using relevant MeSH terms and keywords. Articles were included when they addressed injury prevention, recreational alpine skiing or snowboarding, and musculoskeletal injuries. Two independent reviewers quality assessed all articles meeting inclusion using a validated checklist with higher percentage scores indicating higher quality. Data were subsequently extracted from articles using a standard form and categorized to facilitate data synthesis and interpretation.

Results: Thirty articles met the defined inclusion criteria and scored $72 \pm 17 \%$ (range: $23-100 \%$ ) during quality assessment. From these articles, 80 recommendations for the prevention of musculoskeletal injuries in recreational alpine skiers and snowboarders were sourced, but none pertained to exercise prescription per se. The recommendations were instead grouped into 5 main categories: equipment $(n=24)$, education and knowledge $(n=11)$, awareness and behaviour $(n=15)$, experience $(n=10)$ and third-party involvement $(n=20)$. Most articles $(73 \%)$ contained recommendations relevant to than one of these areas.

Conclusion(s): The importance of targeting physical fitness in injury prevention is accepted in sports medicine and rehabilitation; yet, exercise programs for the prevention of injuries in snow sports are not readily available. As these sports involve high velocities and impact forces, it is not surprising that equipment related injury prevention recommendations were often reported. However, the nature of these sports also indicates a need for appropriate levels of strength, endurance, and conditioning to ensure an adequate control of alpine manoeuvres and avoidance of injuries. Despite the lack of documented exercise programs, the value of using a multi-facetted approach to preventing injuries was evident. The lack of published data in this area is perhaps due to the challenges of performing an exercise-based injury prevention study in recreational skiers and snowboarders, which would need relatively large sample sizes, long-term follow-up, and extensive documentation and attention to confounding variables for valid, reliable and conclusive results.

Implications: Until large-scale experimental research is performed, the prescription of exercises for the prevention of injuries in recreational alpine skiers and snowboarders has limited specificity and depends on empirical evidence from other sports.

Keywords: Injury prevention; Snow sports; Systematic review

Funding acknowledgements: The authors acknowledge the contribution of Maria Hansson, PT, and Mette Bäckström, PT, for their assistance in this work.

Ethics approval: Ethical approval was not required to perform this work.

http://dx.doi.org/10.1016/j.physio.2015.03.3366

Research Report Poster Presentation

Number: RR-PO-10-11-Sat

Saturday 2 May 2015 12:15

Exhibit halls 401-403

\section{A RANDOMISED PLACEBO-CONTROLLED STUDY INVESTIGATING THE EFFECTS OF MOBILISATION TREATMENT DURATION ON PAIN IN PARTICIPANTS WITH CHRONIC LOW BACK PAIN}

$\underline{\text { C. Hebron }}^{1}$, A. Moore ${ }^{1}$, K. Saber Sheikh ${ }^{1}$, A. Jackson ${ }^{2}$

${ }^{1}$ University of Brighton, School of Health Sciences, Eastbourne, United Kingdom; ${ }^{2}$ University of Brighton, Brighton, United Kingdom

Background: A common treatment used by physiotherapists for patients with low back pain is mobilisation. The aim of applying mobilisation treatment is to increase range of movement and reduce pain and stiffness. Therapists choose a specific dose of mobilisation for each patient, which includes a decision on the duration of applied force, commonly up to 3 minutes. Little research has been done to determine the effects of different durations of treatment. There is tentative evidence that increased duration beyond 3 minutes leads to a decrease in pain. 